\title{
Quelles actions au service de la qualité de vie au travail dans les pratiques de management des cadres de santé ?
}

\author{
In : Soins Cadres, n 131, nov. 2021, p. 53-60 \\ DOI : https://doi.org/10.1016/j.scad.2021.10.012
}

Mathieu Malaquin, maître de conférences associé, université Gustave Eiffel, 5, boulevard Descartes, Champs-sur-Marne, 77454 Marne-la-Vallée cedex 2, mathieu.malaquin@univ-eiffel.fr

Pascal Ughetto, professeur de sociologie, université Gustave Eiffel, 5, boulevard Descartes, Champs-surMarne, 77454 Marne-la-Vallée cedex 2, pascal.ughetto@univ-eiffel.fr (06.76.37.37.24) [Auteur de correspondance]

\begin{abstract}
Résumé : De quelle manière les cadres de santé portent-ils des préoccupations de qualité de vie au travail dans leurs pratiques managériales ? A partir d'une étude réalisée dans un département de I'AP-HP, I'article analyse les actions spontanément engagées par ces cadres en invitant à conforter celles qui assurent la « qualité du travail »: il s'agit de consolider ce qui permet d'organiser l'activité des équipes, sans négliger le besoin des cadres de développer à leur tour leur métier.
\end{abstract}

Mots-clés : Qualité de vie au travail ; Cadres de santé ; Management ; Travail

\section{Introduction}

Les conditions de travail reviennent inlassablement dans les préoccupations et les débats internes aux structures médicales et médico-sociales. Elles se situaient au cœur du mouvement infirmier de la fin des années 1980 et du début des années 1990, à partir de termes comparables à ceux qui servent aujourd'hui à faire connaître un épuisement des personnels et leur lassitude vis-à-vis des conditions d'exercice des métiers du soin. Charge de travail, effectifs insuffisants, horaires élevés, mais aussi sentiment de perte de sens; rythmes de travail et encombrement par les actes administratifs au détriment du soin et du rapport aux patients : autant de manifestations de ce qui rend le travail difficile à endurer de la part des équipes soignantes, de la tension entre l'engagement consenti et la fatigue physique et morale éprouvée et d'un sentiment que les conditions de réalisation de l'activité se dégradent (Gheorghiu \& Moatty 2013). A l'image d'autres organisations, publiques ou privées, dans de très nombreux secteurs et d'autres mondes professionnels, les structures hospitalières ont été amenées à ménager une place aux enjeux que l'on désigne désormais à partir de l'expression « qualité de vie au travail »(QVT) (Ughetto 2021). Ces enjeux inspirent aujourd'hui des projets, que les directions d'établissements portent de façon plus ou moins active et qui visent à améliorer le bien-être des personnels (Abord de Chatillon et al. 2017). 
Tout aussi courant est, à l'hôpital ou ailleurs, de s'interroger sur le rôle que peuvent tenir les membres de l'encadrement pour relayer ces actions ou, plus largement, pour intégrer dans leurs préoccupations et leurs pratiques une attention envers les enjeux qu'elles concernent. II est attendu d'eux une attention, une vigilance, à l'égard du sujet autant que la mise en œuvre des plans et dispositifs élaborés par les directions de ressources humaines, de la qualité ou des soins.

Cet article rend compte d'une démarche d'analyse conduite à la demande d'un département médicouniversitaire (DMU) de l'AP-HP. Elle s'est attachée à comprendre ces enjeux à partir de l'activité de ces cadres de santé et la manière dont ces individus investissent leurs tâches. On observe qu'ils se montrent d'ores et déjà soucieux d'agir de façon à contribuer à une qualité du travail de leurs équipes. Loin de devoir construire ex nihilo une sensibilité de ces cadres au thème de la QVT et des pratiques de management y contribuant, il s'agit plutôt de conforter les actions par lesquelles ils tentent déjà de donner à l'activité de leurs équipes de l'organisation et du soutien. Mais cela peut exiger d'accompagner ces cadres pour dans leur réflexivité et leur débat sur leur métier et son rôle dans l'organisation du travail.

\section{Aider les cadres de santé à agir en faveur de la qualité de vie au travail : la demande du DMU et la démarche proposée}

Le DMU dans le cadre duquel l'étude a été réalisée est caractérisé par son souhait de ne pas seulement répondre aux attentes de la direction de l'établissement en matière de QVT mais de porter de façon autonome sa propre sensibilité et ses projets. Attachée à identifier les moyens d'agir, la direction de ce département a estimé que les cadres de santé exerçaient probablement un rôle décisif, qui devait être compris et soutenu pour développer la qualité de vie au travail. Dans le cadre de ses relations avec I'université Gustave Eiffel, elle a exprimé le désir d'être accompagnée dans sa réflexion sur ce rôle des cadres. Quelles pourraient être leurs actions en la matière ? En quoi leurs pratiques de management peuvent-elles intégrer des visées de ce type ? D’ores et déjà, décèle-t-on des actions ou des pratiques qui en relèvent ? La cheffe de service et la cadre supérieure de santé qui assurent la direction de ce DMU ont formulé ces questions auprès d'une promotion de seconde année du master Conduite du changement et sociologie des ressources humaines. La demande concernait des pistes d'action possibles mais avant tout une description et analyse de l'existant.

L'approche proposée a consisté à s'intéresser à l'activité des cadres de santé dans son ensemble pour comprendre quelles actions de qualité de vie au travail ces cadres peuvent déployer au sein de leurs pratiques de management. C'est en comprenant l'activité générale des cadres de santé qu'il est possible de s'interroger sur la place que peuvent y tenir - ou y tiennent déjà - les enjeux d'une qualité de vie au travail des membres de leurs équipes. Il s'agissait de se poser des questions comme : que sont supposés faire les cadres, quelles sont leurs missions ? Quelle activité les voit-on effectivement déployer ? Quels sont les enjeux, les contraintes, les difficultés, mais aussi les ressources ou appuis de cette activité ? Comment les voit-on, dans ce contexte, se confronter à des enjeux de qualité de vie au travail de leurs équipes ? Comment y répondent-ils ? À quelles limites éventuelles se heurtent-ils ? Mais également, comment leur propre activité peut-elle être soutenue?

La crise sanitaire, sa sollicitation hors-normes des personnels soignants et le confinement ont compliqué l'application du protocole envisagé. Néanmoins, l'essentiel de la méthode a pu être conservé. Une enquête qualitative a été effectuée au sein du DMU, auprès des équipes de ses deux sites, de février à mars 2020. Quatorze entretiens (9 en face à face, 5 par téléphone) ont été réalisés par des binômes d'étudiants: 12 avec des cadres de santé, 2 avec des personnels de secrétariat et infirmier. Les entretiens, d'une durée comprise entre une trentaine de minutes et 2 heures 30 , ont été intégralement 
retranscrits et analysés. Ils ont abordé la fonction des cadres de santé et leurs tâches, le service et ses caractéristiques, l'activité de travail déployée, la définition de la qualité de vie au travail et les actions engagées, le parcours personnel des interviewé.es ${ }^{1}$. En outre, 7 observations du travail de cadre et de l'activité du service ont pu être réalisées.

\section{Les cadres de santé du DMU et leur activité}

Cadre de santé : une fonction attendue sur une variété d'actions et une activité très dense

D'une manière générale, les cadres de santé ont à assumer une série de tâches dans des domaines comme la conception et la coordination de l'organisation des soins, la gestion des moyens et de l'information, le management d'équipe, la gestion et le développement des compétences, la gestion des risques et l'animation des démarches qualité, la mise en place et le suivi de projets, etc. De telles tâches demandent aux cadres d'embrasser un large spectre d'actions, certaines interprétées comme de nature administrative, d'autres plus orientées vers le management des individus et des collectifs, d'autres encore renvoyant aux protocoles et à la gestion des risques.

La partie la plus managériale est particulièrement revendiquée. Le suivi des protocoles est moins évoqué par les interviewées mais sans doute perçu comme renvoyant au socle d'expertise normalement attendu de toutes. La partie administrative est parfois perçue comme pesante, mais généralement présentée comme faisant partie intégrante du métier.

Pour les cadres de santé comme dans n'importe quel travail, les tâches à réaliser sont loin de résumer toute l'activité engagée. Les situations professionnelles décrites dans les entretiens et aperçues lors des observations se montrent plus riches qu'une exécution simple et mécanique des tâches. Le début de journée suit généralement un schéma consistant à ouvrir la messagerie avant d'aller saluer les équipes et s'enquérir des urgences et des besoins, avant de revenir au bureau faire des tâches d'ordre administratif. Mais, rapidement, ce schéma peut se complexifier, au gré des circonstances.

Une partie importante du travail des cadres de santé consiste à élaborer de l'organisation, de la planification (plannings des équipes, entrées/sorties des patients...), à se situer dans la prévision, I'anticipation, la mise en adéquation des moyens et des besoins. Parallèlement, toutefois, les réalités de travail consistent à réagir à de nombreux imprévus - urgences, retard d'une ambulance, absence d'un membre de l'équipe... - et à ré-accorder les moyens et les besoins (Bourret 2006 ; Grosjean et Lacoste 1999).

Les cadres de santé savent d'expérience qu'elles doivent compter avec ces impondérables : certains font partie intégrante du métier et de son intérêt; d'autres sont plus difficilement tolérés : « Je me suis vue perdre un après-midi pour des embouts de peak flow. » (Entretien 1)

\section{Des interruptions régulières et un faisceau de contraintes}

Les journées de travail sont constamment parsemées d'interruptions, rompant le cours des actions engagées et nécessitant l'adaptation aux attentes d'une variété d'interlocuteurs. Comme pour d'autres salariés, ces interruptions ont un statut ambivalent : elles peuvent, en renouvelant les situations, donner de l'intérêt au travail ; mais, lorsqu'elles sont vécues comme un flux subi et excessif, elles peuvent être ressenties comme une pénibilité portant atteinte au plaisir pris dans le travail. Leur gestion par les

\footnotetext{
${ }^{1}$ La plupart des personnes interviewées étant des femmes, nous en rendrons compte désormais au féminin.
} 
individus que sont les cadres représente par ailleurs un enjeu d'efficacité du travail collectif et la régulation des sollicitations multiples implique sans doute autant le service dans son ensemble que ces seuls individus.

Ce travail quotidien d'organisation et de réorganisation se réalise à partir d'un faisceau de contraintes à conjuguer. Celles-ci répondent à de nombreux paramètres sur lesquels ces cadres ont un pouvoir variable. Elles concernent souvent la contrainte pesant puissamment sur les moyens, parfois en matériel et, presque toujours, en termes d'effectifs. Dans certains cas, les rotations rapides d'effectifs, la gestion des intérimaires occupent beaucoup du temps des cadres. Les interviewées désignent aussi les logiciels et moyens informatiques plus ou moins souples dans leur utilisation et qui peuvent ralentir le travail. Elles évoquent aussi le cadre architectural : dans l'un des deux établissements, les distances à parcourir sont parfois extrêmement longues :

« C'est très agréable de travailler dans des nouveaux locaux, mais il y a un énorme défaut c'est que [le service] est tout en longueur (...). Et quand on travaille à un bout et que les réserves sont à l'autre bout c'est un peu compliqué. (...) ça coupe un petit peu l'entraide et la communication dans l'équipe. » (Ent. 10)

L'organisation des espaces peut aussi ajouter aux interruptions :

«Les assistantes sociales sont au bout du couloir. On a beau avoir mis un panneau, les gens ne percutent pas et je suis constamment dérangée. » (Ent. 1)

Les configurations propres à chaque service comptent beaucoup. Certains services connaissent une organisation spécifique (par exemple, couplage avec un autre sur un même plateau); d'autres ont un statut symbolique qui leur est propre (une réputation favorable ou négative), attirant plus ou moins les candidats pour occuper les postes. Les patients, l'activité de soin et chirurgicale les concernant, les techniques introduisent d'importantes différences dans les enjeux et les réalités quotidiennes. Tout cela est au cœur de l'activité des cadres de santé et particulièrement porté par elles : I'activité organisatrice et réorganisatrice est quasiment l'essence de leur travail et, selon une expression couramment usitée en ergonomie, il s'agit d'une activité adressée à autrui : les patients, les médecins, divers interlocuteurs, certes, mais avant toute chose les équipes qu'elles encadrent. Cela prépare à l'idée que la qualité de vie au travail de ces équipes est partie intégrante de leur propre activité.

\section{La prise en compte des enjeux de qualité de vie au travail par les cadres du DMU}

Au moment de l'enquête, le DMU a décidé de s'emparer du thème de la qualité de vie au travail depuis déjà plusieurs mois. II l'a inclus dans ses réunions entre services et obtenu un budget à lui consacrer. De fait, lorsque la question leur a été posée, les cadres ont fourni des définitions attestant d'une première appropriation du sujet et renvoyant à une large gamme d'idées.

\section{Les significations associées à la QVT}

Dans certains cas, les définitions ont renvoyé à l'idée d'une articulation à assurer entre le travail et le hors-travail : équilibre vie privée - vie professionnelle, existence de services au personnel (crèches, logements) à tarif préférentiel ou à proximité du lieu de travail, d'autant plus importants dans une ville comme Paris. Les cadres estiment pouvoir y contribuer par des actions comme l'aménagement des plannings en fonction de contraintes individuelles. 
Dans d'autres cas, il était question d'ambiance de travail : bonne entente au sein de l'équipe, bonnes relations avec les médecins, soutien entre collègues. Les cadres peuvent alors s'attribuer une responsabilité en la matière : en pratiquant la bienveillance, en se montrant équitables, en s'attachant à soutenir et accompagner les équipes. Cela renvoie aussi à l'enjeu de créer un sentiment d'appartenance à un collectif, éventuellement en cultivant les moments conviviaux en dehors du travail :

" J'essaye de toujours être bienveillante, d'être à l'écoute, d'être disponible. J'essaye de communiquer avec eux le plus possible. En tout cas, j'essaye d'être présente physiquement le plus possible dans l'équipe. » (Ent. 3)

D’autres définitions font référence à des dispositifs déjà expérimentés ou à tenter : mise à disposition de psychologues, challenges ludiques entre équipes, etc. Il peut également s'agir de l'amélioration des conditions quotidiennes de vie dans le service.

Certaines définitions font porter l'enjeu sur l'existence d'échanges et de réelles communications à l'occasion du travail et sur celui-ci. Elles envisagent de véritables possibilités d'échange entre membres de l'équipe et avec leur encadrement. Les cadres s'assignent alors l'obligation de se rendre disponibles, de se montrer à l'écoute :

" Vous voyez le fait d'être à l'écoute, le fait de faire attention. Je parle beaucoup en individuel, mais aussi dans le service. Et, mon bureau, les agents savent que mon bureau est ouvert, on peut venir ce qui arrive souvent -, on peut venir me voir quand il y a des soucis autant perso' qu'au niveau professionnel. » (Ent. 5)

Enfin, les bonnes conditions d'exécution du travail sont au cœur de certaines conceptions de la QVT. Cela inclut la confiance faite aux équipes et aux individus et les latitudes qui leur sont laissées dans l'organisation de leur propre travail, la mise à disposition d'un matériel de bonne qualité, des espaces de travail facilitant la réalisation des tâches.

Dans tous les cas, disposer d'un effectif de personnel moins contraint et d'une charge de travail moins lourde apparaît comme une condition élémentaire.

Les formes actuelles de prise en charge actuelle de la qualité de vie au travail

Dans leur activité, et spécialement dans leurs pratiques de management, les cadres du DMU semblent d'ores et déjà se montrer attentives à des enjeux de qualité de vie au travail. De fait, le développement d'une qualité de vie au travail ne relève pas que du futur mais fait déjà partie du DMU, où l'on observe :

- des actions et des dispositifs : tai chi, groupe de parole animé par des psychologues...

- des efforts pour développer l'interconnaissance à l'échelle du DMU (récemment constitué par l'intégration de plusieurs services), mais qui s'avèrent apparemment encore compliqués à mener à leur terme; néanmoins, des groupes de discussion s'organisent sur des réseaux sociaux, en particulier par le biais de WhatsApp; certains services disposent déjà de pratiques telles qu'un repas de fin d'année pris en commun ;

- une responsabilité que s'assignent fréquemment les cadres d'être joignables et à l'écoute, notamment des individus, y compris en dehors du temps de travail ; 
- en matière d'exercice du travail, un soin des cadres à assurer la transmission de l'information, à fluidifier la communication, à organiser les échanges. Ce sont là des actions moins visibles mais qui participent nettement de la qualité des conditions d'exercice du travail.

\section{Appréhender la QVT à partir des enjeux de qualité du travail}

Les pratiques de management revendiquées par les cadres du DMU et leurs définitions de la QVT insistent sur les enjeux conjoints de qualité du service réalisé et de qualité des conditions offertes aux équipes pour l'accomplissement de leur travail. Les propos tenus dans les interviews ne sont pas sans faire écho à un débat, au sein des sciences sociales, quant à la définition pertinente de la qualité de vie au travail. Un psychologue comme Yves Clot est connu pour défendre l'importance d'une réflexion centrée sur la qualité du travail et ses conditions de possibilité (Clot et Lallement 2015). II souligne que les approches les plus courantes de la qualité de vie au travail n'abordent guère l'enjeu du contenu du travail. N'interrogeant pas l'organisation du travail, elles ne favorisent pas la réflexion sur les conditions d'un travail bien fait, la possibilité donnée par l'organisation aux individus de faire un travail de qualité. On peut ainsi analyser les actions existantes ou possibles du DMU en mettant en lumière celles qui portent le plus spécifiquement sur le travail et à ses conditions d'exercice, sans nécessairement négliger celles qui se situeraient à des niveaux plus périphériques.

\section{Les cadres du DMU d'ores et déjà sensibles aux conditions d'exercice du travail}

Bien que situés en périphérie de la réalisation du travail lui-même, certains enjeux identifiés au sein du DMU ont, en effet, leur importance : la qualité de l'articulation entre temps professionnels et temps hors-travail, à travers les questions du transport, du logement, des crèches. Les concernant, le DMU a sans doute peu de moyens d'action mais sa direction peut imposer fréquemment le sujet dans les instances appropriées.

Les cadres semblent, par ailleurs, partager la préoccupation d'améliorer l'interconnaissance, notamment dans un contexte qui fait cohabiter les services de deux sites : identifier ses homologues, savoir qui contacter, se transmettre de l'expertise. Susciter cette interconnaissance entre équipes et entre cadres eux-mêmes paraît constituer un socle pour toute autre initiative à entreprendre. Les outils numériques de communication ont probablement leur rôle à jouer dans ce domaine, en rebondissant sur la tendance spontanée de collectifs à se doter de groupes sur les réseaux sociaux. Ils complètent une connaissance mutuelle se nourrissant aussi d'échanges informels et de moments conviviaux.

Si ce sujet n'est pas problématisé spontanément par les interviewées du point de vue des conditions de réalisation du travail, il n'en recouvre pas moins un enjeu de ce point de vue, qui est de structurer et faire vivre des collectifs : collectifs de métier ou autour d'une fonction commune, mais aussi liens effectifs entre soignants et non-soignants (on peut penser au rôle fondamental du travail de secrétariat et à son intégration à part entière dans le collectif), entre médecins et reste de l'équipe soignante. Cela ne se substitue pas à l'existence, par ailleurs, de collectifs de cadres qui leur soient propres.

Au sein du travail lui-même, la communication entre individus et entre équipes, la transmission des informations sont identifiées dans de nombreux entretiens comme jouant un rôle clef dans la fluidité et l'efficacité du travail collectif, la sérénité qui y préside, l'ambiance coopérative de travail. Beaucoup de choses sont portées, en la matière, par les cadres, à travers la disponibilité dont ils ou elles cherchent à faire preuve et leur effort de communication des informations. 
"Parce que ça c'est très important, pour une équipe, de savoir où [les infirmières et les aidessoignantes] vont, qui elles accueillent et comment et quand. C'est important de leur fournir les informations et qu'elles ne cherchent pas, (...) qu'elles soient pas seules au monde à se dépatouiller. » (Ent. 6)

On voit ici comment les cadres de santé envisagent l'action qui peut être la leur dans des termes qui relèvent d'efforts pour assurer une qualité du travail de leurs équipes, en faisant en sorte que le travail ne s'exerce pas dans un désordre source de démobilisation mais, au contraire, dans un environnement organisé. Certains services ont, cependant, plus que d'autres, poussé la réflexion sur les possibilités de mettre en place une organisation pour assurer cela et ne pas le faire reposer sur les seuls cadres : courtes réunions hebdomadaires limitant la place accordée à l'information descendante au profit d'échanges; postes spécifiques d'infirmiers de coordination et d'apprentissage. Certains interviewés mentionnent l'intérêt de mettre en place des livrets d'accueil aidant les nouveaux occupants d'un poste d'encadrement à se repérer dans le service, dans les tâches, dans les ressources. Le DMU, a-t-il été proposé à l'issue de la recherche, pourrait en faire un chantier de réflexion pour étendre ou susciter de tels dispositifs.

Les espaces de travail et les matériels entrent également dans le sujet des conditions d'exercice du travail. Ils sont souvent considérés comme une donnée et sont subis. En la matière, les services sont inégaux : certains ont bénéficié de locaux et de matériels récents, souvent appréciés positivement ; d'autres demeurent avec des matériels anciens voire dépassés, des locaux non rénovés. Même avec des locaux récemment revus, des contraintes spatiales peuvent s'exprimer: manque de luminosité, configuration mal pensée, distances importantes à parcourir. "On est en plein milieu, on n'a pas de fenêtre sur rue, on est vraiment dans un bocal, quoi. » (Ent. 9). Sans nier la difficulté à intervenir a posteriori sur des aménagements figés dans l'architecture retenue, certains sujets de conditions spatiales d'exercice de l'activité pourraient néanmoins être débattus par le DMU. C'est du moins l'une des conclusions de la recherche, en s'appuyant sur l'idée que l'espace, tout en étant largement déjà là et contraignant par nature, fait aussi toujours l'objet d'efforts des individus pour le mettre à leur main (Lave 1988). Par exemple, sur la base d'une analyse de l'activité, il est envisageable de rapprocher des activités qui entretiennent de fréquentes interactions ou de doter le DMU d'équipements de déplacement (vélos, trottinettes).

Certains logiciels sont salués par les interviewées pour leur pertinence et leur facilité d'utilisation, d'autres sont blâmés pour leur utilisation très contraignante. Ces sujets peuvent, à leur tour, être portés par la direction du DMU dans les instances décisionnaires concernées. Cela n'exclut pas de réfléchir, au sein du département, à organiser de la transmission et du partage d'expérience autour des outils.

Les modes et styles de management comptent.

Les interviews évoquent, directement ou non, les modes et styles de management. De fait, ceux-ci contribuent de façon décisive au développement de pratiques de coopération à l'intérieur des services. Les entretiens décrivent des situations contrastées, allant de services où l'ambiance d'ensemble est perçue comme positive et apaisée et où médecins et membres de l'encadrement font confiance et discutent des réalités du travail, à des services héritant de fonctionnements plus compliqués : plus grande difficulté de communication quotidienne entre les équipes infirmières et aides-soignantes et les équipes médicales ou à l'intérieur de chacune d'entre elles. Ces fonctionnements peuvent comporter une dimension systémique, avec un jeu d'acteurs difficile à transformer. 
Leur fonction amène les cadres de santé à jouer le rôle d'un agent de liaison entre les médecins et les équipes infirmières et aides-soignantes et de gardiens de la bonne ambiance de travail, sans être les seuls à même d'y aider :

"C'est moi qui suis au milieu. Je suis au milieu de ça et c'est mon rôle de gérer ces conflits-là. J'essaie d'appliquer des choses mais, après, les médecins, ils sont là aussi, ils arrivent à calmer les choses, à relativiser et, après, ça se passe bien. » (Ent. 5)

Développer un chantier relatif à la qualité de vie au travail ne semble pas rencontrer de difficulté dans le premier cas. Dans le second, les cadres peuvent se sentir en difficulté : comment évoquer le sujet sans risquer l'échec, l'incompréhension ou la mise en cause?

"Pouvoir avoir ce mot - "qualité de vie au travail" - et vous avez devant vous quelqu'un qui est épuisé, vous vous dites: "est-ce que vous vous foutez de lui ?", c'est un peu s'exposer aux foudres de la personne. » (Ent. 9).

Peut-être l'accompagnement de la direction du DMU est-il d'autant plus nécessaire pour aider ces cadres à en faire une occasion de travailler simultanément la QVT et l'amélioration des communications internes et de la coopération.

\section{Poursuivre et amplifier les actions entreprises en adoptant systématiquement une approche en termes de qualité du travail}

En conclusion de l'analyse, le DMU a été invité à poursuivre et amplifier ce qu'il avait d'ores et déjà entrepris tout en resserrant son approche sur une lecture systématique des enjeux de QVT en termes de qualité du travail (voir encadré).

\section{Des cadres de santé attentifs à l'organisation de l'activité de travail}

Il lui a été suggéré que cela peut impliquer :

- des actions et des dispositifs aidant à organiser le travail : le DMU peut appuyer toute volonté des collectifs de travailler sur les outils, les informations, l'adaptation des règles formelles et informelles, la clarification des rôles, etc. L'analyse du travail par les cadres joue alors un rôle essentiel, comme leur capacité à transformer une obligation (par exemple, effectuer des retours d'expérience sur les événements indésirables) en occasion de mieux comprendre le travail réel ;

- des initiatives et des processus pour consolider les collectifs : consolider l'interconnaissance est utile mais ne représente pas un but en soi. Plus fondamentalement, il s'agit de faire en sorte que celle-ci renforce les coopérations, le partage des interprétations des problèmes et l'élaboration commune de réponses à ceux-ci.

Un rôle spécifique incombe, dès lors, à la direction du DMU envers les cadres. Il peut consister à :

- stimuler l'analyse de la part de ces cadres sur le diagnostic spécifique de leur secteur, l'activité de leurs équipes, les actions qui mériteraient d'être entreprises ;

- soutenir particulièrement les cadres exerçant dans des services où des chantiers relatifs à la qualité de vie au travail dépendent préalablement ou simultanément d'une réinstallation de meilleures conditions de coopération et de communication. 
Une condition : soutenir l'activité des cadres et les conditions d'exercice du métier

Plusieurs entretiens ont été l'occasion d'indiquer que les cadres de santé elles-mêmes méritaient que I'on s'intéresse à leurs conditions de travail. L'analyse des entretiens suggère que cela pourrait se faire en se montrant attentif à deux points en particulier.

a) L'extrême connexion des cadres : par souci d'anticiper sur la journée à venir, la charge de travail, les actions à mener, les problèmes à régler, presque toutes les interviewées ont déclaré se connecter à leurs mails avant leur arrivée au travail, lors du trajet, au petit-déjeuner ou même au réveil. Sans porter atteinte aux marges de manœuvre et à l'organisation personnelle qu'elles s'attachent à construire par ce moyen dans le but de maîtriser leur travail et sa charge, le sujet de la surconnexion et des possibilités d'aménagement d'une déconnexion paraitrait volontiers pouvoir donner lieu à une réflexion collective, à partir d'une analyse de l'activité, de ses enjeux, de ses ressources.

b) Le métier de cadre lui-même : beaucoup d'interviewées livrent une compréhension de leur rôle de cadres comme impliquant de soulager au maximum les équipes d'une série de complications ou de contraintes, voire de prendre soin des équipes et des individus, de chercher à "les arranger ». Les entretiens soulignent, pour la valoriser, l'importance d'une empathie avec les équipes d'autant plus fréquente qu' « on a tous été infirmiers ou infirmières. »

«Mon rôle c'est vraiment de faire en sorte que le personnel soit dans de bonnes conditions de travail. » (Ent. 2)

« Je suis en astreinte $\mathrm{H} 24$, c'est-à-dire qu'on peut m'appeler sur mon portable perso le matin, à 5 heures, on peut m'appeler. On m'appelle tout le temps. Ça, après, c'est un inconvénient pour moi parce que, même le week-end, pendant les vacances je reçois des SMS, des appels de toute l'équipe. » (Ent. 5)

Ces pratiques incitent à ne pas aborder la QVT comme une simple préoccupation gestionnaire, répondant à des objectifs de conformité réglementaire, mais comme s'ancrant dans des formes de vie, des cadences de travail, des astreintes que les individus peuvent s'imposer spontanément, tenus qu'ils se sentent d'honorer les exigences de leur travail. Cela conduit aussi ces cadres à se sentir obligées de porter individuellement la responsabilité de la bonne santé physique et mentale des membres de leurs équipes, au risque d'hériter à leur tour du burn out qu'elles souhaitent épargner à ces derniers.

Les cadres semblent beaucoup envisager leur métier comme se situant à part entière dans la communauté des soignants (" De la femme de ménage jusqu'à la directrice de l'hôpital, pour moi, on est tous ensemble, c'est comme ça qu'on arrive à faire marcher » (Ent. 1). Cette perception offre l'avantage d'un management s'exerçant en se sentant pleinement concernées par le travail et la vie des équipes mais c'est au risque d'une difficulté à s'assumer dans un rôle spécifique de managers, appelées à exercer plus en surplomb. De fait, dans les institutions hospitalières ou ailleurs, les cadres exercent une fonction qui les place au contact des situations que traitent leurs équipes mais ces individus incarnent aussi les logiques d'organisation (Ughetto 2017, 2018). Cela les oblige, selon les termes d'une cadre interrogée, à « faire le deuil des soins », ce qui, au regard des entretiens, s'avère plus ou moins facile :

«Savoir prendre des décisions, dire aux autres "Attends, [ce que tu as fait ne convient pas]...", ce positionnement peut être compliqué à avoir, surtout au départ. » (Ent. 1). 
Il semblerait donc qu'aider les cadres à discuter collectivement des réalités d'un travail qui les amène à être à la fois impliqués dans la communauté soignante et ne plus être seulement membres de cette communauté pourrait s'avérer utile.

Si les cadres interrogées saluent l'effet de groupes de parole pour les membres de leurs équipes, ellesmêmes peuvent avoir besoin d'espaces qui leur soient spécifiquement consacrés. Leur fonction serait de leur permettre d'échanger sur les problèmes qui sont typiques de leur fonction, de débattre de leurs causes et conséquences, d'élaborer des réponses, de se doter d'outils, de formuler des propositions à leur propre hiérarchie. L'organisation de tels espaces de discussion n'a pas été spontanément envisagé par le DMU et serait toutefois à réfléchir.

\section{Conclusion}

Que peut-on demander aux cadres de santé de porter en matière de qualité de vie au travail ? La question mérite, pour être traitée, de distinguer une approche large de la QVT - qui inclut des sujets relativement périphériques relativement aux conditions de réalisation de l'activité - et une problématique de la qualité du travail, sur laquelle l'accent doit être mis. Dans une " enveloppe » étendue, l'articulation des temps personnels et professionnels ou l'ambiance de travail traitée de façon très générale ainsi que l'interconnaissance constitueront des points à ne pas négliger. Mais le cœur de ce que les personnels soignants s'efforcent d'exprimer et de faire débattre sans relâche depuis trois décennies concerne véritablement le travail et les conditions dans lesquelles ils doivent l'effectuer. C'est donc la qualité du travail qui importe. L'étude conduite dans le cadre du DMU évoqué dans cet article montre que les cadres de santé agissent souvent dans ce domaine. Ces actions méritent d'être confortées. II s'agit notamment d'actions visant la qualité des collectifs de travail et l'organisation de l'activité. II semble important d'aider ces cadres à agir de façon systématique dans cette direction : en organisant les communications, le partage de l'information, l'élaboration continuelle des règles de travail pertinentes; en considérant les espaces de travail et les matériels non comme de l'intendance mais en s'attachant à en faire de véritables instruments du travail des équipes. Sur le plan des styles de management, les plus à même de soutenir la qualité du travail semblent être celles qui donnent aux personnels des latitudes pour s'organiser et qui reposent sur la confiance.

\section{Bibliographie}

Abord de Chatillon E., Blondet C., Branche, B., Crambes C., Commeiras N., Malaquin M., Rossano M. et Valette A. La prévention des risques psychosociaux : le cas du secteur hospitalier (public et privé). Observations de terrain et analyses à partir de l'enquête Conditions de travail 2013, Etudes, recherche et débat, n 4, DGAFP, fév. 2017.

Bourret P. Les cadres de santé à l'hôpital : un travail de lien invisible, Seli Arslan 2006.

Clot Y. et Lallement M. Qualité de vie au travail et qualité du travail. La revue des conditions de travail, 2015 ; 3 : 45-56.

Gheorghiu MD. \& Moatty F. L'hôpital en mouvement. Changements organisationnels et conditions de travail, Liaisons 2013.

Grosjean M. et Lacoste M. Communication et intelligence collective : le travail à l'hôpital. Le Travail humain, PUF, 1999.

Lave J. Cognition in Practice : Mind, mathematics and culture in everyday life, Cambridge University Press, 1988. 
Ughetto P., Line managers as work professionals in the era of workplace health professionalization. In Beyond Safety Training: Embedding Safety in Professional Skills, Bieder C., Gilbert P., Journé B., Laroche H., eds., Springer, 2017: 23-33.

Ughetto P. Les cadres, acteurs de la prévention et de la santé au travail : à quelles conditions ? Archives des Maladies Professionnelles et de l'Environnement, 2018 : 79(3), 271.

Ughetto P. La qualité de vie au travail au risque de sa transformation en process. In: Les servitudes du bien-être au travail, Le Garrec S., Erès, 2021: 169-188.

Encadré. Inciter les cadres de santé à agir en faveur de la qualité du travail et les soutenir dans leur activité

Articulation des temps personnels et professionnels

Interconnaissance et ambiance de travail

Des actions et

des dispositifs aidant à organiser le travail.

Favoriser I'analyse du travail
Collectifs de travail et conditions de réalisation du travail :

$>$ Communications, informations, règles de travail : à organiser

$>$ Espaces de travail et matériels : en faire de véritables instruments

$>$ Modes et styles de management : favoriser les pratiques managériales reposant sur la confiance et donnant des latitudes pour s'organiser
Des

initiatives

et des

processus

pour

consolider

les

collectifs

Pour les cadres :

- Stimuler leur analyse et diagnostic de leur secteur, de l'activité de leurs équipes, des actions à entreprendre ;

- Soutenir celles et ceux qui exercent dans des services où communication et coopération sont à faire évoluer :

- Soutenir les conditions d'exercice du métier : surconnexion / déconnexion ; articulation entre l'implication dans la communauté soignante et le fait d'endosser les logiques d'organisation; espaces de discussion entre cadres. 